operative Nervus-radialis-Läsion, zusammen mit der Exposition gegenüber Wasser und Irritantien im Haushalt ein chronischirritatives Ekzem der betroffenen Finger entwickelte. Die Sensibilisierung gegenüber Calendula erfolgte vermutlich während der Anwendung nach der früheren Schulteroperation. Die erneute Applikation führte zu einer allergischen Reaktion, die durch die Okklusion mit Gummifingerlingen verstärkt wurde.

\section{Sonstige allergische Krankheitsmanifestationen}

\section{P35 Pollenassoziierte Nahrungsmittelallergie mit Gelenkbeteiligung - spezifische Immuntherapie mit den Kreuzallergenen}

\section{R. Bless ${ }^{1}$, T. Fröhlich ${ }^{2}$}

${ }^{1}$ Allergologische Praxis. St.-Hedwig-Klinik, Mannheim; ${ }^{2}$ Pulmologischallergologischer pädiatrischerQualitätszirkel Heidelberg/Mannheim

Einführung: Pollenassoziierte Nahrungsmittelallergien mitGelenkbeteiligung sind ein seltenes und in der Literatur kaum beschriebenes Ereignis. (Übersicht in: Metcalfe D, Simon R. Food Allergy, Wiley-Blackwell, 2003, S. 541 ff.) Mit vorliegender Kasuistik möchten wir auf Besonderheiten und „Fallstricke“ dieses Krankheitsbildes hinweisen.

Kasuistik: 5 Jahre alter Junge, welcher seit dem Säuglingsalter unter atopischem Ekzem leidet - zusätzlich heftigen urtikariellen Reaktionen auf Tomate und Zitrusfrüchte. Im Verlauf zusätzlich obstruktive Bronchitiden im Frühjahr. Mit 1 1/2 Jahren erstmals Anaphylaxie nach Erdnussaufnahme. Ab dem 3. Lebensjahr zunehmende bds. Hüftgelenksbeschwerden, wiederholt gedeutet als "Coxitis fugax" mit zahlreichen stationären Aufnahmen unter der Verdachtsdiagnose „Poststreptokokkennephritis und juvenile idiopathische Arthritis“.

Diagnostik: Stark erhöhter Antistreptokinase-Titer: 20480 U/l, Antistreptokokken DNAse B über 2400U/l; Gesamt-IgE: 1110 kU/l; IgE Rast Birke: 100 AU/l; IgE Rast Birke rBet v1: 74,3 AU/l; IgE Rast Birke rBet v2: neg.; IgE Rast Erdnuss 24,4 AU/l; IgE Rast Birke Ara h 5: 20,5 AU/l.

Verlauf: Da es deutliche Hinweise auf einen Beschwerdegipfel während des Birkenpollenfluges gab, entschlossen wir uns zu einer spezifischen Immuntherapie (SIT) mittels der kreuzreagierenden Birken- und Haselnusspollen. Homologe Allergene in Birkenpollen und Lebensmitteln: Bet v 1: Haselnuss, Bet v 2 (Birkenpollenprofilin): Erdnuss, Haselnuss, Tomate (Ara h 5).

Therapie: SIT mit Allergenextrakt 50\% Birke, 50\% Haselnuss. Nach abgeschlossener SIT rückläufige Beschwerden bezüglich der pollenassoziierten Symptome. Als Erfolgskontrolle nach einer SIT wurde die Messung der spezifischen IgG-AK herangezogen, welche in unserem Fall deutlich angestiegen waren: IgG Rast Birke 2,8 ug/ml +++, IgG Rast Erdnuss 2,6 ug/ml +++, IgG Rast Haselnuss 3,8 ug/ml +++.

Schlussfolgerung: Bei einem nachgewiesenem pollenassoziierten Allergiesyndrom stellt die SIT mit den kreuzreagierenden Pollen eine erfolgversprechende Therapieoption dar.
P36 Persistierende Infekte sind häufige Triggerfaktoren der chronischen Urtikaria im Kindesalter

D. Wieczorek, A. Kapp, B. Wedi

Klinik und Poliklinik für Dermatologie und Venerologie der Medizinischen Hochschule Hannover

Hintergrund: Chronische Urtikaria (CU) ist eine häufige Erkrankung in der Gesamtbevölkerung, dagegen machen Kinder einen nur geringen Anteil aus (Inzidenz 2,1 und 6,7 bzw. $3,4 \%$ ). Hier fließen jedoch alle Urtikariaformen mit ein, also auch die viel häufigere akute Urtikaria und physikalische Formen der Urtikaria. Untersuchungen zu Triggerfaktoren und Therapieerfolgen existieren kaum.

Patienten und Methoden: Mithilfe eines standardisierten diagnostischen Vorgehens wurde die Häufigkeit verschiedener Triggerfaktoren und der Erfolg entsprechender Therapieoptionen bei CU im Kindesalter untersucht. Hierzu wurden 40 Kinder mit einer CU zwischen 6 Mo. und 17 J. einbezogen. Ergebnisse: Bei Kindern mit CU wurde eine atopische Diathese nicht häufiger als bei der übrigen Bevölkerung vorgefunden. Besonders häufig zeigten sich serologische Hinweise auf subklinische, bakterielle Infekte (ASTA 11/40, ASL 14/40, $\alpha$ DNaseB 20/40), weiterhin erhöhte Leukozyten (4/40) und auffälliges CRP (2/40), aber auch eine persistierende Yersiniose (3/33) konnte serologisch nachgewiesen werden. Ergänzend führten wir die Bestimmung der Titer von Virusantikörpern, z. B. EBV und CMV (1/6), sowie die Stuhluntersuchung auf pathogene Keime (2/10) durch. In einigen Fällen konnten wir mittels Urease-Atemtest bzw. Stuhltest Infektionen mit Helicobacter pylori (6/31) nachweisen. In seltenen Fällen konnten mittels autologem Serumtest (8/22) oder Bestimmung von Schilddrüsen-Auto-Ak (3/31) Hinweise für eine autoreaktive Urtikaria diagnostiziert werden.

Schlussfolgerung: Grundsätzlich scheinen sich die Triggerfaktoren bei Kindern nicht von Erwachsenen zu unterscheiden. In unserer Untersuchung waren persistierende, oft unbemerkte chronische Infekte (v. a. mit Streptokokken und Staphylokokken) bei Kindern insgesamt die häufigste Ursache für das Auftreten der CU. In den meisten Fällen war insgesamt zusätzlich zur spezifischen Therapie die Gabe von Antihistaminika indiziert, oft in erhöhter Dosierung.

\section{Allergische Berufserkrankungen}

\section{P37 Bedeutung der tertiären Prävention am Beispiel eines Metzgers mit Proteinkontaktdermatitis}

\section{U. Krüger, I. Röhrig, H. Petering}

Dermatologische Gemeinschaftspraxis, Hildesheim

Die Proteinkontaktdermatitis wurde vor 30 Jahren erstmals beschrieben. Sie tritt als berufsbedingte Dermatose in der Nahrungsmittelindustrie auf. Tierische und pflanzliche Proteine gelten als Auslöser. Therapeutisch steht die Meidung des auslösenden Agens im Vordergrund.

Ein 38-jährigen Metzger, der seit 21 Jahren in der Produktion und im Verkauf tätig ist, stellte sich mit lividen, ju- 